\title{
61. NEW ESTIMATES OF COMETARY DISINTEGRATION TIMES AND THE IMPLICATIONS FOR DIFFUSION THEORY
}

\author{
O. V. DOBROVOL'SKIJ \\ Institute of Astrophysics, Dushanbe, U.S.S.R.
}

\begin{abstract}
Numerical methods are used to establish the fraction of solar radiation utilized in the sublimation of ice on the surface of a cometary nucleus. An estimate can then be made of the lifetime of the nucleus. For a nucleus of radius $2 \times 10^{5} \mathrm{~cm}$ in an orbit of semilatus rectum $0.6 \mathrm{AU}$ the lifetime is 300 revolutions. Comparison with diffusion theory reveals a significant deficiency of the theory in the case of comets with small reciprocal semimajor axes.
\end{abstract}

The rate of disintegration of comets and its relation to the statistics of cometary orbits is of great importance to the cosmogony of comets.

Since an icy model is the most probable one, that is what we shall discuss (Rijves, 1970). The lifetime $\tau$ of a comet can conveniently be determined in terms of the efficiency of the solar radiation $\eta$, defined as the ratio of the amount of solar radiation expended in disintegration to the total amount of solar energy incident to the nucleus. The mass $\mathrm{d} M$ lost by the nucleus during time $\mathrm{d} t$ will be

$$
\mathrm{d} M=-\frac{Q_{0} r_{0}^{2}}{L r^{2}} s \mathrm{~d} t=-\frac{Q_{0} r_{0}^{2} s}{L c} \eta \mathrm{d} v
$$

where $Q_{0}$ is the solar constant, $r_{0}$ the astronomical unit, $r$ the heliocentric distance, $s$ the cross-section area of the nucleus, $L$ the specific heat of disintegration, $c$ the constant of the law of areas, and $v$ the comet's true anomaly. If the nucleus is a sphere of density $\delta$, the change in the nuclear radius $R$ will be

$$
\mathrm{d} R=-\eta \frac{Q_{0} r_{0}^{2}}{4 c L \delta} \mathrm{d} v
$$

In one revolution about the Sun the initial radius $R_{0}$ will decrease to

$$
R=R_{0}-\frac{2 \pi Q_{0} r_{0}^{2}}{4 c L \delta} \bar{\eta}
$$

$\bar{\eta}$ being the efficiency of the solar radiation averaged over the true anomaly.

We assume that after $n$ revolutions $R$ will diminish to zero; this number is then given by

$$
n=\frac{2 c L R_{0} \delta}{\pi Q_{0} r_{0}^{2} \bar{\eta}}
$$

Hence the total lifetime $\tau$ of the comet (in years) is

$$
\tau=\frac{K}{\bar{\eta}} a^{3 / 2} p^{1 / 2}
$$


where $a$ is the semimajor axis and $p$ the semilatus rectum (both measured in AU), and $K$ is the dimensionless quantity

$$
K=\frac{2 \delta L R_{0}\left(\gamma M_{\odot} r_{\odot}\right)^{1 / 2}}{\pi Q_{0} r_{0}^{2}},
$$

$\gamma$ being the gravitational constant and $M_{\odot}$ and $r_{\odot}$ the mass and radius of the Sun.

Expressing $r$ in AU, we may with reasonable precision write $\eta$ in the form

$$
\eta=\gamma_{10} r^{-\alpha}
$$

then

$$
\bar{\eta}=\frac{\eta_{0}}{p^{\alpha}} J_{\alpha}
$$

where

$$
J_{\alpha}=\frac{1}{\pi} \int_{0}^{\pi}(1+e \cos v)^{\alpha} \mathrm{d} v
$$

$e$ being the orbital eccentricity; and obviously $J_{0}=J_{1}=1$. For $\alpha \geq 2$ we have the recurrence formula

$$
J_{\alpha}=\frac{2 \alpha-1}{\alpha} J_{\alpha-1}-\frac{\alpha-1}{\alpha}\left(1-e^{2}\right) J_{\alpha-2}
$$

Thus, within the range of validity of the approximation (4) we have

$$
\tau=\frac{K}{\eta_{0}} \frac{a^{3 / 2} p^{\alpha+1 / 2}}{1+k_{\alpha} e^{2}}
$$

where $k_{\alpha}$ is given in Table $\mathrm{I}$.

TABLE I

$k_{\alpha}$ as a function of $\alpha$

\begin{tabular}{ll}
\hline$\alpha$ & $k_{\alpha}$ \\
\hline 0 & 0 \\
1 & 0 \\
2 & $1 / 2$ \\
3 & $5 / 6$ \\
4 & $13 / 12$ \\
\hline
\end{tabular}

Various determinations of $\alpha$ have been made for an $\mathrm{H}_{2} \mathrm{O}$ nucleus. For instance, Markovich and Tulenkova (1968) and Dobrovol'skij (1966) found that when $r \geqslant 1 \mathrm{AU}$, $\alpha=3 / 2$ and $\eta_{0} \approx 1 / 2$, while for smaller values of $r$, the coefficient $\eta$ approaches 1 asymptotically. Thus, if the perihelion distance $q \geqslant 1 \mathrm{AU}$, we shall have, approximately,

$$
\tau=2 K a^{3 / 2} p^{2} .
$$


If $q<1$, this may be regarded as the lower limit of $\tau$, although when $p<0.63$ AU the lower limit is more suitably given by

$$
\tau_{\min }=K a^{3 / 2} p^{1 / 2} \text {. }
$$

Taking $L=2.7 \times 10^{10} \mathrm{erg} \mathrm{g}^{-1}$, we shall have, for a nucleus with $R_{0}=2 \times 10^{5} \mathrm{~cm}$ and $\delta=1 \mathrm{~g} \mathrm{~cm}^{-3}$,

$$
K \approx 500
$$

so that, when $p=0.6 \mathrm{AU}, \tau \approx 300$ revolutions.

According to Shtejns (1961), diffusion theory gives the number of comets $\nu$ passing perihelion annually and having reciprocal semimajor axes $z=1 / a$ in the range $(z, z+1)$ as

$$
\nu_{z}=A \exp \left(-g z^{1 / 2} \tau^{-1 / 2}\right),
$$

where $A$ and $g$ are constants. Shtejns has discussed Equation (12) for $\tau=7$ and 60 revolutions. Figures 1 and 2 show distributions with $\tau=7,60$, and 300 revolutions, the constant $A$ having been selected in each case so as to satisfy the points given by Shtejns (1961).

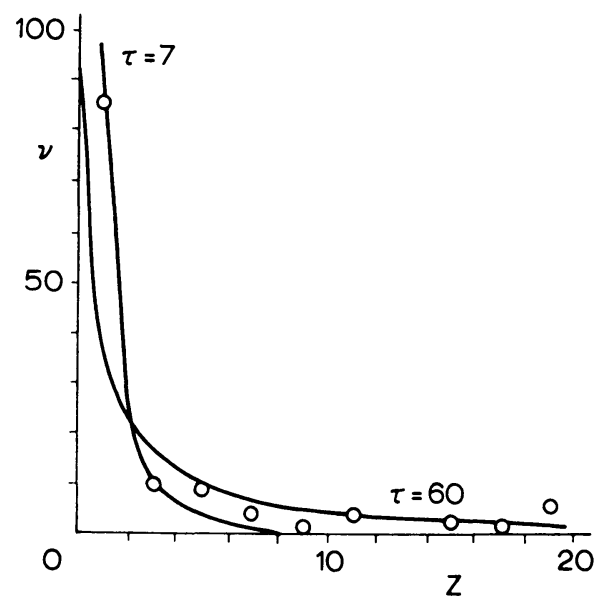

Fig. 1. Distribution of the number of comets $\nu$ as a function of reciprocal semimajor axis $z$ for lifetimes $\tau=7$ and 60 revolutions. Observed points are denoted by circles.

Calculated curves for $\tau=7$ and 60 revolutions coincide qualitatively with the points given by Shtejns; for $\tau=7$ the fit is best for small $z$, while for $\tau=60$ large $z$-values are represented more closely. For $\tau=300$ revolutions a satisfactory description can be given only in the case of large $z$. Comets with small $z$ do not fit the theoretical relationship at all: we could choose $A$ so that the curve would pass through the first point of the observed distribution, but then it would not correspond to any of the others, and no reasonable variation of $q$ would help eliminate the discrepancy.

We conclude that Equation (12) does not agree with the observations and should be 
replaced, or else the diffusion theory should be developed more rigorously, with account taken in particular of the dependence of $\tau$ on $a$ in the original diffusion equation.

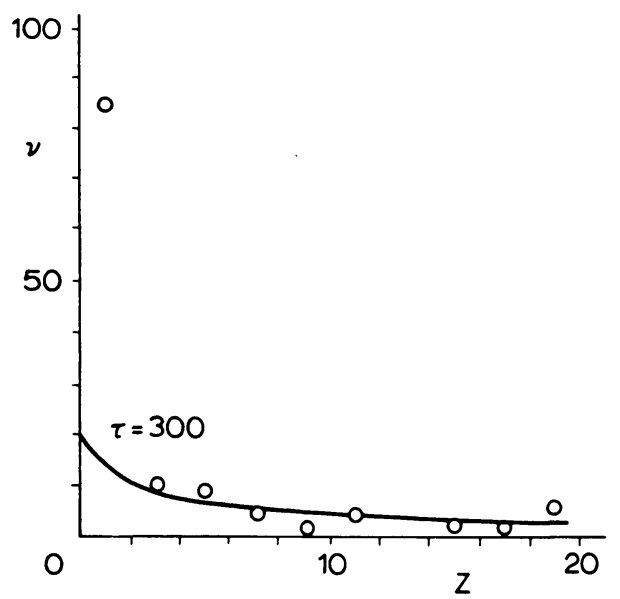

Fig. 2. Distribution of $\nu$ as a function of $z$ for $\tau=300$ revolutions.

\section{References}

Dobrovol'skij, O. V.: 1966, Komety, Nauka, Moscow.

Markovich, M. Z. and Tulenkova, L. N.: 1968, Probl. Kosmich. Fiz. No. 3, 25.

Rijves, V. G.: 1970, Komety i Meteory No. 17, 3.

Shtejns, K. A.: 1961, Astron. Zh. 38, 107. 